\title{
Oscillatory electroosmotic flow in a microchannel with slippage at the walls
}

\author{
O. Bautista, M. Peralta, J. Arcos
}

\begin{abstract}
In this work, we conduct a theoretical analysis of an oscillatory electroosmotic flow in a parallel-plate microchannel taking into account slippage at the microchannel walls. The governing equations given by the Poisson-Boltzmann (with the Debye-Hückel approximation) and momentum equations are nondimensionalized from which four dimensionless parameters appear: an Reynolds angular number, the ratio between the zeta potentials of the microchannel walls, the electrokinetic parameter and the dimensionless slip length which measures the competition between the Navier slip length and the half height microchannel. The principal results indicate that the slippage has a strong influence on the magnitude of the oscillatory electroosmotic flow increasing the velocity magnitude up to $50 \%$ for the numerical values used in this work.
\end{abstract}

Keywords - electroosmotic flow, slippage, microchannel

\section{Introduction}

Many devices used in microfluidics have attracted considerable interest because of their potential for use in novel applications in various areas of science and industry. Biotechnology is a continuously growing field and has given rise to many experiments and applications involving microelectro-mechanical systems (MEMSs) [1], such as the separation of deoxyribonucleic acids (DNA) [2], the separation of proteins [3], mixing processes [4] and the detection of bacteria or viruses [5]. Operations involving MEMS devices frequently require the pumping, control and manipulation of samples. To drive fluids through such devices, one can use electrokinetic effects [?] such as electroosmosis, which refers to the induction of a liquid flow via the application of an external electric field along electrostatically charged surfaces. Electroosmotic flows (EOFs) can be used for various tasks, such as dispersion control in micromixing based on the field effect [?], to create a nonuniform zeta potential along the channel walls, thereby achieving improved electrokinetic separation [6]. In applications involving separation and mixing in microchannels, oscillatory electroosmotic flows (EOFs ) driven by alternating currents (AC-driven EOFs) have become increasingly popular because of their broad applications and marked advantages compared with steady EOFs (DC-driven EOFs). Another important factor that strongly affects the dynamic behavior of DC- and AC-driven EOFs is the fact that microchannels are typically fabricated with different wall materials; in practice, diverse materials can modify the zeta potentials at the microchannel walls [7]. In many of the works cited above, the analysis was performed under the assumption of zeta potentials that are different at the different walls but uniform in the longitudinal direction of the microchannel, a scenario that can be created by imposing an external DC electric field. However, there are no studies in which an analysis of an EOF has been performed for AC-driven EOFs with different wall zeta potentials and slippage at the walls. This analysis is expected to serve as a benchmark for extending our understanding of the spatiotemporal analysis of microfluidic devices for which precise knowledge of transient transport phenomena is important. In addition, this investigation has practical implications for the ability to exploit in future works various combinations of separation and mixing effects in microchannels.

\section{Mathematical Formulation}

\section{A. Physical Model}

The physical model under study is depicted in Fig. 1. We consider the periodic oscillatory electroosmotic flow (OEOF) of a symmetric (z:z) electrolyte solution in a parallel-plate microchannel of height $H$ and length $L$, such that $L \gg H$; here, is the valency of the electrolyte. The origin of the coordinate system is defined to lie at the left corner of the lower surface of the microchannel. We consider that the walls of the channel can have different (asymmetric) zeta potentials, that is, $\zeta_{1} \neq \zeta_{2}$, and that the high concentration of electric charges is localized near the channel walls, within the EDL, whose thickness is represented by $\kappa^{-1}$, where $\kappa=\left(2 e^{2} z^{2} n_{\infty} \varepsilon k_{B} T\right)^{1 / 2}$ [8]; here $e, n_{\infty}$, $\varepsilon, k_{B}$ and $T$ represent the elementary charge, the ionic number concentration in the bulk solution, the dielectric permittivity of médium, the Boltzmann constant and the absolute temperature. The electroosmotic flow is caused by the movement of the electric charges adjacent to the walls of the microchannel when an external periodic electric field, given by $E_{x}(t)=E_{0} \sin (\omega t)$, is applied; here, $t, \omega$ and $E_{0}$ represent time and the angular frequency and amplitude of the sinusoidal electric signal, respectively. It is assumed that the flow is laminar and that the electric double layers on the inner surfaces of the microchannel do not overlap, that is, $H \gg \kappa^{-1}$. The ends of the microchannel are assumed to be at the same pressure, $P_{0}$.

\section{O. Bautista and J. Arcos}

Instituto Politécnico Nacional. SEPI-ESIME Azcapotzalco, Av. De las Granjas 682, Col Santa Catarina, 02250, México Ciyt, Mexico.

\section{Peralta}

Universidad Autónoma Metropolitana-Iztapalapa, División de Ciencias Básicas e Ingeniería, Av. San Rafael Atlixco 186, Col. Vicentina, 09340, México City, México. 


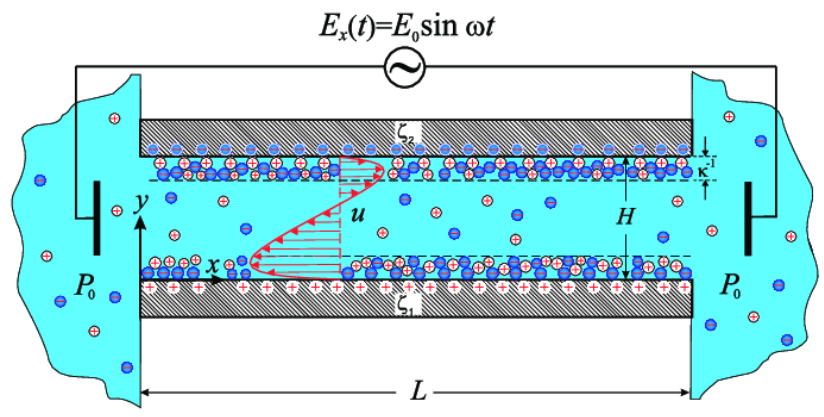

Fig. 1. Schematic diagram of the physical model under study.

\section{B. Governing Equations}

Electric potential. The electric potential distribution close to the inner surface of the capillary is governed by the Poisson-Boltzmann equation [8]. Under the Debye-Hückel approximation, and considering that $L \gg H$, such equation becomes $\square$

$$
\frac{d^{2} \psi}{d y^{2}}=\kappa^{2} \psi
$$

Equation (1) is subject to the following boundary conditions:

$$
\psi=\zeta_{1} \text { at } y=H \quad \text { and } \quad \psi=\zeta_{2} \text { at } y=-H
$$

Flow field. To determine the hydrodynamic of the OEOF, we assume that the flow is unidirectional and fully developed, with $u(y, t)$ representing the velocity in the $x$ direction. In the absence of a pressure gradient, we use the modified NavierStokes equation

$$
\rho \frac{\partial u}{\partial t}=\mu \frac{\partial^{2} u}{\partial t^{2}}+\rho_{e} E_{x}(t)
$$

where $\rho$ and $\mu$ are the mass density and viscosity of the fluid, respectively. Equation (3) is subject to the following boundary conditions:

$$
u=\mp b \frac{\partial u}{\partial y} \text { at } y= \pm H
$$

In the above equation, $b$ denotes the Navier length. Note that we focus the analysis on long times, i.e., when the transient stage has elapsed and the flow field is only periodic. Therefore, the initial condition is not needed to solve the momentum equation.

\section{Non-dimensional formulation and analytical solution}

We define the dimensionless variables $\bar{y} \equiv y / H, \bar{t} \equiv t / t_{c}, \bar{\psi}$ $\equiv \psi / \psi_{c}$, and $\bar{u} \equiv u / u_{\mathrm{HS}}$, where $\psi_{\mathrm{c}}=\mathrm{k}_{\mathrm{B}} \mathrm{T} / \mathrm{ze}, \mathrm{u}_{\mathrm{HS}}=-\zeta 1 \mathrm{E} 0 / \mu$ is the Helmholtz-Smoluchowski velocity Masliyah [8], and $t_{c}=$ $H^{2} / v$, where $v=\mu / \rho$ represents the kinematic viscosity of the fluid. Therefore, the dimensionless Poisson-Boltzmann equation, Eq. (1), is given by

$$
\frac{d^{2} \bar{\psi}}{d \bar{y}^{2}}=\bar{\kappa}^{2} \bar{\psi},
$$

where $\bar{\kappa} \equiv \kappa H$. The boundary conditions associated with Eq. (5) are given in dimensionless form as $\bar{\psi}=\bar{\zeta}_{1}$ at $\bar{y}=1$, and $\bar{\psi}=\bar{\zeta}_{1}$ at $\bar{y}=-1$. Here, $\bar{\zeta}_{1}=\zeta_{1} z e / k_{B} T$ and $\bar{\zeta}_{2}=\zeta_{2} z e / k_{B} T$. The solution of Eq. (5), is given by

$$
\begin{aligned}
\bar{\psi}(\bar{y})=\left(\frac{\bar{\zeta}_{1}+\bar{\zeta}_{2}}{4 \cosh (\bar{\kappa})}+\frac{\bar{\zeta}_{1}-\bar{\zeta}_{2}}{4 \cosh (\bar{\kappa})}\right) \exp (\bar{\kappa} \bar{y}) \\
+\left(\frac{\bar{\zeta}_{1}+\bar{\zeta}_{2}}{4 \cosh (\bar{\kappa})}-\frac{\bar{\zeta}_{1}-\bar{\zeta}_{2}}{4 \cosh (\bar{\kappa})}\right) \exp (-\bar{\kappa} \bar{y})
\end{aligned}
$$

The dimensionless version of the momentum equation, Eq. (3), is written as follows:

$$
\operatorname{Re}_{\omega} \frac{\partial \bar{u}}{\partial \bar{t}}=\frac{\partial^{2} \bar{u}}{\partial^{2} \bar{y}}+\bar{\kappa}^{2} \bar{\psi} \sin \bar{t}
$$

where $R e_{\omega}=\omega H^{2} / v$ represents the angular Reynolds number [9] and denotes the competition between the characteristic diffusion time and the characteristic time associated with the oscillatory electric field. The dimensionless boundary conditions needed to solve Eq. (7) are:

$$
\bar{u}( \pm 1, \bar{t})=\left.\mp \gamma_{s} \frac{\partial \bar{u}}{\partial \bar{y}}\right|_{\bar{y}= \pm 1}
$$

where $\gamma_{s}=b / H$ denotes the ratio between the Navier length and the microchannel half height. To obtain the time-periodic solution of the velocity, we define the complementary complex velocity $\hat{u}$ in such a manner that $\bar{u}(\bar{y}, \bar{t})=$ $\operatorname{Im}[F(\bar{y}) \exp (i \bar{t})]$, where $\operatorname{Im}$ denotes the imaginary part of the complementary complex velocity. Therefore, substituting the proposed solution into Eq. (7), and solving the resulting differential for $F(\bar{y})$, we obtain

$$
\begin{gathered}
F(\bar{y})=B_{1} \exp \left(\sqrt{i R e_{\omega} \bar{y}}\right)+B_{2} \exp \left(-\sqrt{i R e_{\omega} \bar{y}}\right) \\
+\frac{\bar{\kappa}^{2}}{i R e_{\omega}-\bar{\kappa}^{2}}\left[A_{1} \exp (\bar{\kappa} \bar{y})\right. \\
\left.+A_{2} \exp (-\bar{\kappa} \bar{y})\right] .
\end{gathered}
$$

The expressions for $\mathrm{B}_{1}$ and $\mathrm{B}_{2}$ are given by

$$
\begin{aligned}
& B_{1} \\
& =-\frac{1}{2} \frac{\bar{\kappa}^{2}}{i R e_{\omega}-\bar{\kappa}^{2}}\left\{\left(A_{1}\right.\right. \\
& \left.+A_{2}\right) \frac{\cosh (\bar{\kappa})+\gamma_{s} \bar{\kappa} \sinh (\bar{\kappa})}{\cosh \left(\sqrt{i R e_{\omega}}\right)+\gamma_{s} \sqrt{i R e_{\omega}} \sinh \left(\sqrt{i R e_{\omega}}\right)} \\
& \left.+\left(A_{1}-A_{2}\right) \frac{\sinh (\bar{\kappa})+\gamma_{s} \bar{\kappa} \cosh (\bar{\kappa})}{\sinh \left(\sqrt{i R e_{\omega}}\right)+\gamma_{s} \sqrt{i R e_{\omega}} \cosh \left(\sqrt{i R e_{\omega}}\right)}\right\}
\end{aligned}
$$




$$
\begin{aligned}
& B_{2} \\
& =\frac{1}{2} \frac{\bar{\kappa}^{2}}{i R e_{\omega}-\bar{\kappa}^{2}}\left\{\left(A_{1}\right.\right. \\
& \left.-A_{2}\right) \frac{\sinh (\bar{\kappa})+\gamma_{s} \bar{\kappa} \cosh (\bar{\kappa})}{\sinh \left(\sqrt{i R e_{\omega}}\right)+\gamma_{s} \sqrt{i R e_{\omega}} \cosh \left(\sqrt{i R e_{\omega}}\right)} \\
& \left.-\left(A_{1}+A_{2}\right) \frac{\cosh (\bar{\kappa})+\gamma_{s} \bar{\kappa} \sinh (\bar{\kappa})}{\cosh \left(\sqrt{i R e_{\omega}}\right)+\gamma_{s} \sqrt{i R e_{\omega}} \sinh \left(\sqrt{i R e_{\omega}}\right)}\right\} .
\end{aligned}
$$

In Eqs. (9)-(11), the parameter A1 and A2 are defined as follows:

$$
A_{1}=\left(\frac{\bar{\zeta}_{1}+\bar{\zeta}_{2}}{4 \cosh (\bar{\kappa})}+\frac{\bar{\zeta}_{1}-\bar{\zeta}_{2}}{4 \cosh (\bar{\kappa})}\right)
$$

and

$$
A_{2}=\frac{\bar{\zeta}_{1}+\bar{\zeta}_{2}}{4 \cosh (\bar{\kappa})}-\frac{\bar{\zeta}_{1}-\bar{\zeta}_{2}}{4 \cosh (\bar{\kappa})}
$$

From Eqs. (9)-(11), when $\gamma_{s}=0$, the dimensionless velocity profile under the no-slip condition is recovered, which has been previously published [10].

\section{Results}

For all numerical calculations and for estimating the dimensionless parameters involved in the analysis, we select a suitable combination of typical values of physical parameters of EOFs as follows: $0.1 \leq H \leq 15 \mu \mathrm{m} ; 1 \leq \kappa^{-1} \leq 500 \mathrm{~nm} ; \rho^{\sim} 10^{3}$ $\mathrm{kg} \mathrm{m}^{-3} ;\left|\zeta_{1}\right|,\left|\zeta_{2}\right| \leq 25 \mathrm{mV}$; and $0.1 \leq \omega \leq 100 \mathrm{rad} \mathrm{s}^{-1}$. In what follows, dimensionless velocity and concentration profiles are shown.

Dimensionless velocity profiles $\bar{u}$ as a function of the dimensionless transversal coordinate $\bar{y}$, and evaluated at arbitrary values of the dimensionless time $\bar{t}$ are shown in Fig. 2. Here, in Figs. 2(a) and 2(b) we show velocity fields, for $\gamma_{s}=0$ (no slip condition) and for $\gamma_{s}=0$, respectively. We assume a relatively small value of the angular Reynolds number $R_{\omega}=0.1$, which means that the inertial effect is small as compared against the viscous and electric forces, as can be deduced from Eq. (7). It is evident that in this limit, the term on the left-hand side of such equation can be neglected, and under this condition, the asymptotic behavior of the velocity is $\bar{u} \sim H(\bar{y}) \sin (\bar{t})$, meaning that the velocity is in-phase with the external electric field. This result has been previously explained by Peralta et al. [10] and therefore, details are omitted. In should be noted that in Ref. [10] the slippage was not considered in the analysis. In the present case, by comparing Figs. 2(a) and 2(b), is noted that the slippage increases the velocity magnitude up to $50 \%$ for the assumed values of the involved parameters in the analysis. Another aspect that should be observed is that using the relatively small value of $R_{\omega}=0.1$ the velocity profiles are uniform through the cross-section of the microchannel, which is typical when the mirochannel height is large compared with the Debye length, i.e., $\bar{\kappa} \gg 1$.

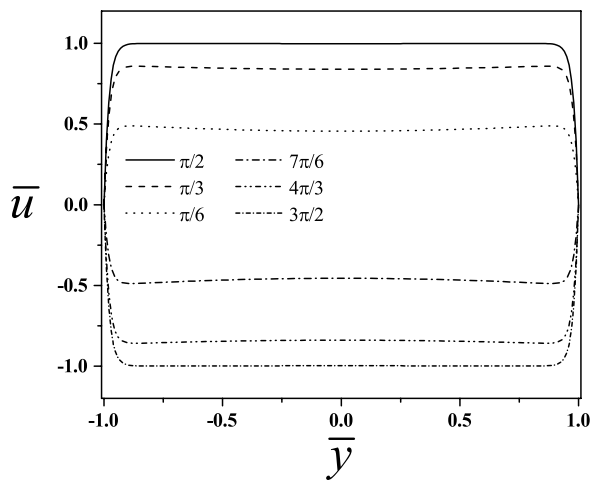

(a)

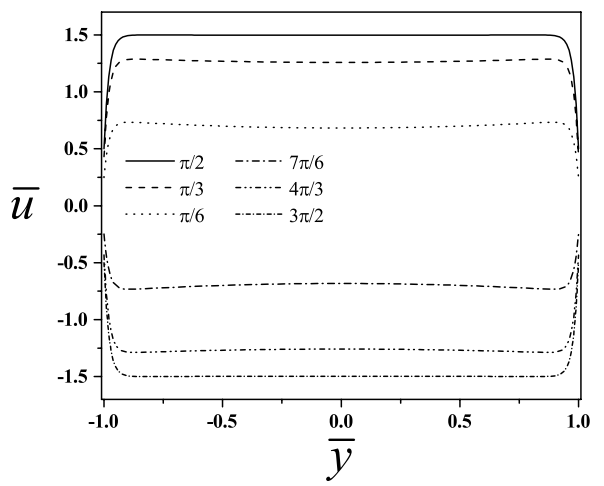

(b)

Fig. 2. Dimensionless velocity profiles evaluated at different values arbitrary of the dimensionless time $\bar{t}$. Here, $R_{\zeta}=1, \bar{\kappa}=50, R_{\omega}=0.1$ with (a) no slip condition, $\gamma_{S}=0$; and (b) with a value of the dimensionless slip length $\lambda=0.01$.

In Figs. 3 we show the effect of the parameter $R_{\omega}$ on the dimensionless velocity profile for a condition of the slippage of $\gamma_{s}=0.01$. Here, a relatively large value of $R_{\omega}=100$ is used. As can be seen, for this condition, large velocity gradients are present only close to the microchannel walls, and they remain uniform in the middle part of the cross-section. This means that in contrast to Fig. 2, here, the inertial effect is strong and confined to a boundary layer close to the walls. It is important to mention that the effect of the slip condition at the wall can be seen at $\bar{y}=0$ and at $\bar{y}=1$.

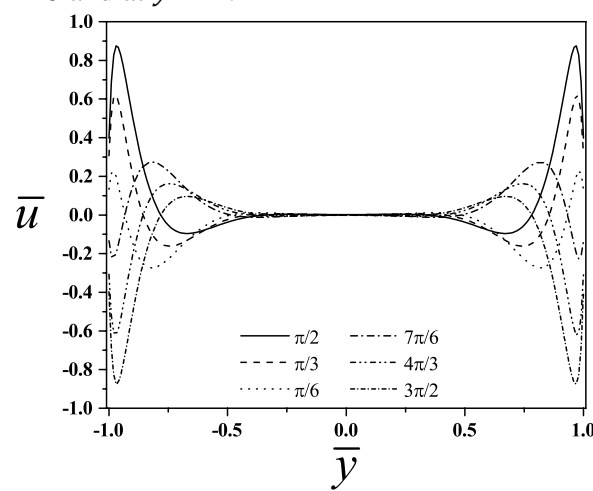

Fig. 3 Dimensionless velocity profiles evaluated at different values arbitrary of the dimensionless time $\bar{t}$. Here, $R_{\zeta}=1, \bar{\kappa}=50, R_{\omega}=100$ and $\gamma_{s}=$ 0.01 . 
In Fig. 4 we show the simultaneous effect of the slip condition and the asymmetry of the zeta potentials at the walls. In this figure, the asymmetry of the zeta potentials is represented by the value of $R_{\zeta}=-1$. This means that one of the walls is positively charged and the another with a negative charge. In this case, the velocity profiles are asymmetric due to the electric force in the electric double layer act in opposite direction. We could anticipate that the simultaneous effect of the asymmetry of the zeta potentials and the slippage at the walls could be used for enhancing micromixing or species separation in microchannels if desired. Such a study has been previously conducted but with the assumption of a constant external electric field $[10,11]$ but no slip condition at the walls.

\section{E. Conclusions}

In this work, we conducted the theoretical analysis of an oscillatory EOF, where slippage at the walls was assumed. In addition, the analysis takes into account asymmetric zeta potentials. It is shown that the dimensionless velocity magnitude is increased when the slip condition is assumed in comparison with the case of no slippage. Also, it was shown the effect of the angular Reynolds number on the dimensionless velocity profiles, which modifies notably the hydrodynamic behavior.

Future work consists of carrying out a similar analysis to that conducted here for non-Newtonian fluids and considering different types of the oscillatory electric fields. In addition, the transport of a neutral solute under the hydrodynamic conditions included in the present work will be carried out.

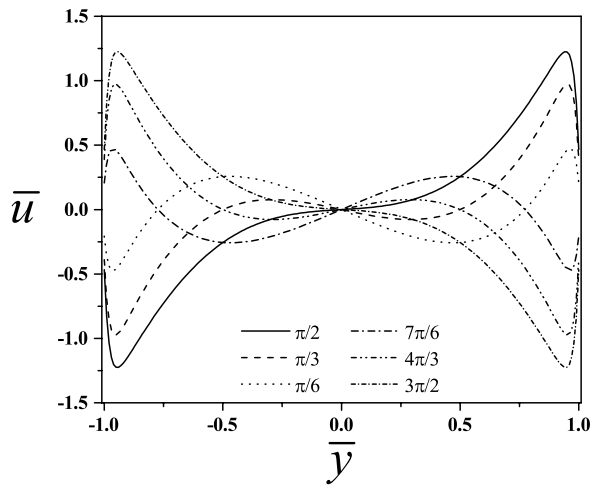

Fig. 4 Dimensionless velocity profiles evaluated at different values arbitrary of the dimensionless time $\bar{t}$. Here, $\mathrm{Rz}=-1, \mathrm{k}=50, \mathrm{Rw}=100$ and $\lambda=0.01$.

\section{Acknowledgment}

This work was supported by the Fondo Sectorial de Investigación para la Educación through research Grant Nos. CB-2013/220900 from SEP-CONACYT and 20180768 from SIP-IPN in Mexico.

\section{References}

[1] N. T. N. S. Shaegh, N. Kashaninejad, D. T. Phan, Design, fabrication and characterization of drug delivery systems based on lab-on-a-chip technology, Adv. Drug. Deliv. Rev. 65 (2013) 1403-1419.

[2] C. River, H. Lee, A. Hirsch, S. Hamilton, Microfluidics for medical diagnostics and biosensors, Chem. Eng. Sci. 66 (2011) 1490-1507.

[3] T. Yasui, M. R. Mohamadi, N. Kaji, Y. Okamoto, M. Tokeshi, Y. Bada, Characterization of low viscosity polymer solutions for microchip electrophoresis of non-denatured proteins on plastic chips, Biomicrofluidics 4 (2011) 044114.

[4] G. Whitesides, A. Stroock, Flexible methods for microfluidics, Phys. Today 54 (6) (2001) 42-46.

[5] M. R. P. Gascoyne, J. Satayavivad, Microfluidic approaches to malaria detection, Acta Trop. 89 (2004) 357-369.

[6] B. Kirby, E. H. Jr., Zeta potential of microfluidic substrates: 1. theory, experimental techniques, and effects on separation., Electrophoresis 25 (2004) 187-202.

[7] K. Huang, Z. Hong, J. Chang, Microfluidic mixing on application of traveling wave electroosmosis, Eur. J. Mech. B. Fluids 48 (2014) 153164.

[8] J. Masliyah, S. Bhattacharjee, Electrokinetic And Colloids Transport Phenomena, John Wiley \& Sons, 2005.

[9] L. G. Leal, Advanced Transport Phenomena. Fluids Mechanics and Convective Transport Processes, Cambridge University Press, 2007.

[10] M. Peralta, J.Arcos, F. Méndez, O. Bautista, Oscillatory electroosmotic flow in a parallel-plate microchannel under assymetric zeta potentials, Fluid Dyn. Res. 49 (2017) 035514.

I. Medina, M. Toledo, F. Méndez, O. Bautista, Pulsatile electroosmotic flow in a microchannel with asymmetric wall zeta potentials and its effect on mass transport enhancement and mixing, Chem. Eng. Sci. 184 (2018) 259-272.

[11] E. K. Zholkovskij, J. H. Masliyah, and A. E. Yaroshchuk, Broadening of neutral analyte band in electroosmotic flow through slit channel with different zeta potentials of the walls, Microfluid. Nanofluid. 15 (2013), 35-47. 